Article

\title{
Residual Stress Distribution and Microstructure at a Laser Spot of AISI 304 Stainless Steel Subjected to Different Laser Shock Peening Impacts
}

\author{
Wenquan Zhang, Jinzhong Lu* and Kaiyu Luo \\ Received: 28 October 2015; Accepted: 18 December 2015; Published: 25 December 2015 \\ Academic Editor: Hugo F. Lopez \\ School of Mechanical Engineering, Jiangsu University, Zhenjiang 212013, China; \\ nicequan1991@163.com (W.Z.); kyluo@ujs.edu.cn (K.L.) \\ * Correspondence: jzlu@mail.ujs.edu.cn; Tel.: +86-511-8879-7198; Fax: +86-511-8878-0352
}

\begin{abstract}
The effects of different laser shock peening (LSP) impacts on the three-dimensional displayed distributions of surface and in-depth residual stress at a laser spot of AISI 304 stainless steel were investigated by X-ray diffraction technology with the $\sin ^{2} \varphi$ method and MATLAB 2010a software. Microstructural evolution in the top surface subjected to multiple LSP impacts was presented by means of cross-sectional optical microscopy (OM) and transmission electron microscopy (TEM) observations. Experimental results and analysis indicated that residual stress distribution and microstructure at a laser spot depended strongly on the number of multiple LSP impacts, and refined grain and ultra-high strain rate play an important role in the improvement of compressive residual stress of AISI 304 stainless steel. The analysis of treatment of the extended surface was presented to obtain uniform surface properties on the top surface of AISI 304 stainless steel.
\end{abstract}

Keywords: multiple laser shock peening; residual stress distribution; microstructure; ultra-high strain rate

\section{Introduction}

Laser shock peening (LSP) is a kind of advanced surface enhancing method which employs the mechanical effect of laser shock wave induced by a laser beam to improve the fatigue resistance, damage tolerance, corrosion resistance of metallic materials and alloys. More and more researchers have been paying comprehensive attention to LSP due to its high-pressure (in the scale of GPa), high-energy (more than $1 \mathrm{GW} / \mathrm{cm}^{2}$ ), ultrafast (several tens of nanoseconds) and ultra-high strain rate (more than $10^{6} \mathrm{~s}^{-1}$ ) [1-3].

Over the last two decades, substantial research efforts have yielded significant insights into the effects of overlapping LSP treatment and multiple LSP impacts on the mechanical properties and microstructure of metallic materials and alloys. For example, Wang et al. investigated the effects of overlapping LSP impacts on stress corrosion behavior of 7075 aluminum alloy laser welded joints, and results showed that the elongation, time of fracture and static toughness were improved by $11.13 \%, 20 \%$ and $100 \%$ after overlapping LSP impacts, respectively [4]. Trdan et al. confirmed the insignificant influence of LSP without coating treatment direction on micro-hardness distribution, indicating essentially homogeneous conditions in both longitudinal and transverse directions [5]. Jia et al. presented that repeated LSP impacts have a very beneficial effect on surface hardening, and the high-cycle fatigue life of LSPed Ti834 alloy increases due to the fact that the introduction of compressive residual stress can delay the initiation and growth of the fatigue crack [6]. Correa et al. established a good correlation between simulations, residual stress measurements and fatigue life of 
A12024-T351 specimens treated by LSP [7]. In our previous work, we reported that the improvement of the stress corrosion cracking (SCC) resistance of AISI 304 stainless steel was caused by compressive residual stress and grain refinement after massive LSP impacts [8]. We also indicated that typical microstructure and residual stress in the surface layer of LY2 aluminum alloy [9] and AISI 304 stainless steel have a close relation with the number of multiple LSP impacts. A single LSP impact can refine the original grain in the near-surface layer mainly by mechanical twins (MTs) in a single direction and multiple LSP impacts can refine the original grain mainly by MTs in multi-directions, which are the direct reason why LSP can improve the nano-hardness, elastic modulus, and residual stress of AISI 304 stainless steel [10]. When laser shock wave acts on the surface layer, local plastic deformation occurs in the LSPed region and the affected region. These investigations mainly focused on the interaction of adjacent LSP impacts during overlapping LSP treatment and multiple LSP impacts. In practice, the surfaces of metallic components are often overlappingly treated by multiple LSP treatment. Uneven residual stress distribution on the component surface often deteriorates the surface properties of the treated component [11], and the uniformity of residual stress in the surface layer of metallic component after massive LSP impacts depends strongly on residual stress distribution and microstructure at a laser spot. However, over the years, only few researchers have investigated how the influence mechanism of a single LSP impact and the number of multiple LSP impacts on residual stress distribution at a laser spot affect the uniformity of surface properties on the extended surface.

This paper aimed to investigate the effects of different LSP impacts on surface and in-depth residual stress distributions of AISI 304 stainless steel at a laser spot. The three-dimensional residual stress displayed distribution as a function of the number of multiple LSP impacts was highlighted. In particular, microstructure in the top surface under different LSP impacts is characterized and analyzed. Finally, the underlying influence mechanism of typical substructure induced by laser shock wave on residual stress distribution at a laser spot was determined and we also analyzed how the underlying influence mechanism affects the surface properties' uniformity on the extended surface. These topics can provide some insight into the uniformity of compressive residual stress generated by massive overlapping LSP impacts, which has a lot of practical use in engineering application.

\section{Experimental Procedures}

\subsection{Specimen Preparation}

AISI 304 austenitic stainless steel was chosen in this study, and its chemical composition was $0.06 \mathrm{C}, 1.54 \mathrm{Mn}, 18.47 \mathrm{Cr}, 0.30 \mathrm{Mo}, 8.3 \mathrm{Ni}, 0.37 \mathrm{Cu}, 0.48 \mathrm{Si}, 0.027 \mathrm{Nb}$ and balance Fe (wt. \%). The mechanical properties of AISI 304 stainless steel are shown in Table 1 [12]. All specimens from the same steel sheet were cut into dimensions of $5 \mathrm{~mm} \times 5 \mathrm{~mm} \times 2 \mathrm{~mm}$ (width $\times$ length $\times$ thickness). Before LSP treatment, all specimen surfaces were progressively mechanically polished using SiC paper with grit numbers from 150 to 1800 (including 150\#, 360\#, 600\#, 800\#, 1200\# and 1800\#) to achieve smooth surfaces. Subsequently, these specimens were cleaned with acetone and subjected to ultrasonic vibration to degrease their surfaces.

Table 1. Mechanical properties of AISI 304 austenitic stainless steel.

\begin{tabular}{cc}
\hline Type & Value \\
\hline Tensile strength, $\left(\sigma_{\mathrm{b}}\right)\left(\mathrm{kgf} / \mathrm{mm}^{2}\right)$ & 520 \\
Specific gravity, $(d)\left(\mathrm{g} / \mathrm{cm}^{3}\right)$ & 7.93 \\
Yield strength, $(\sigma)\left(\mathrm{kgf} / \mathrm{mm}^{2}\right)$ & 205 \\
Vickers-hardness $(\mathrm{HV})$ & 200 \\
Elongation, $(\delta)(\%)$ & 40 \\
\hline
\end{tabular}




\subsection{LSP Experiment}

LSP experiments were carried out by a Q-switched Nd: YAG laser produced by Thales Company in Paris, France with a wavelength of $1064 \mathrm{~nm}$ at Laser Technology Institute in Jiangsu University. For this application, the laser spot diameter was $3 \mathrm{~mm}$, and a pulse laser with a duration of $10 \mathrm{~ns}$ and a pulse energy of $9.3 \mathrm{~J}$ was used as LSP energy source. The output beam of single flat-top laser pulse was attenuated and sent to a charge-coupled-device (CCD) camera produced by SVC ASSET MANAGEMENT INC in California, CA, United States, and its profile can be seen in Figure 1. To ensure the mechanical effect of laser shock wave, professional aluminum tape produced by $3 \mathrm{M}$ company in St Paul, MN, USA, with a thickness of $100 \mu \mathrm{m}$ was used as an ablation medium for plasma initiation, and a water film with a thickness of 1-2 $\mathrm{mm}$ was set as a transparent confining layer. To investigate the effects of the number of LSP impacts on microstructure and residual stress distribution at a laser spot, three types of LSPed specimens were prepared in the present study, and these specimens were treated by different LSP impacts at a single laser spot. According to the number of LSP impacts, we defined these specimens subjected to one LSP impact, two LSP impacts, and three LSP impacts as single LSP impact, dual LSP impacts, and triple LSP impacts, respectively.
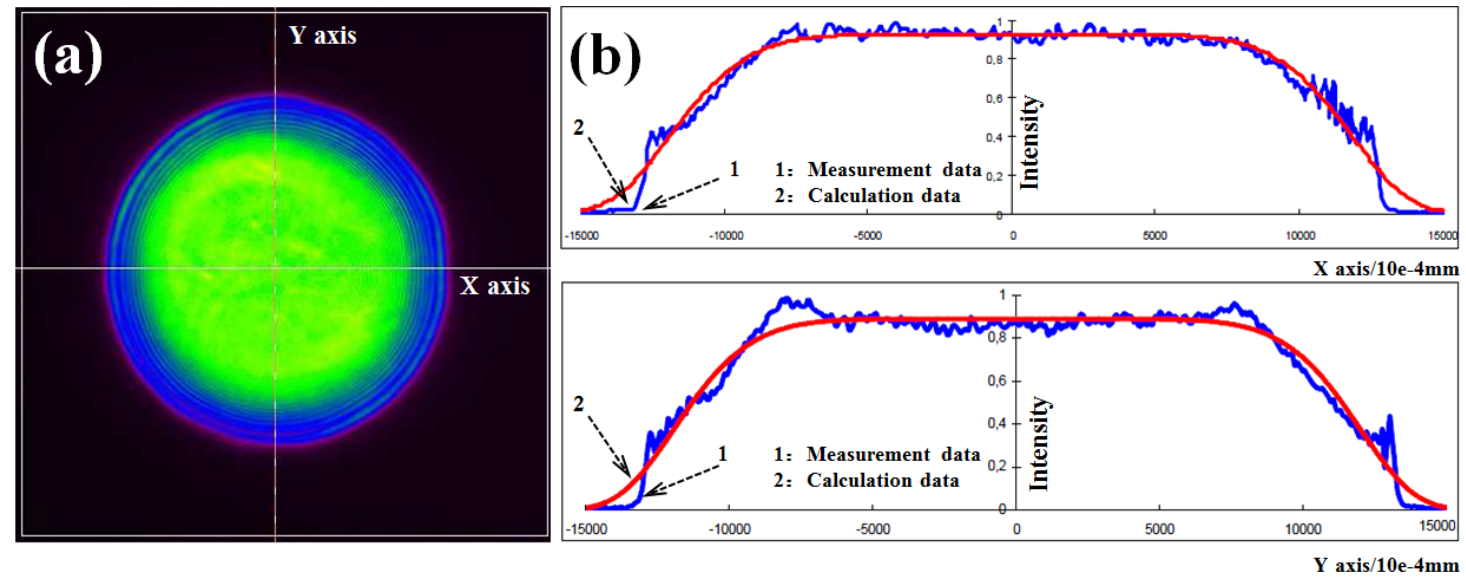

Figure 1. The output beam of single flat-top laser pulse and corresponding spatial profiles along horizontal and perpendicular directions. perpendicular direction.

(a) Along horizontal direction; (b) along

\subsection{Residual Stress Measurement and Microstructural Observations}

Surface residual stresses of all specimens were measured by X-ray diffraction technology with the $\sin ^{2} \varphi$ method [13]. The X-ray beam diameter was about $2 \mathrm{~mm}$, and the $X$-ray source was the $\mathrm{Cr}-\mathrm{K} \alpha$ ray. The feed angle of the ladder scanning was $0.1^{\circ}$ per second, and the scanning starting angle and terminating angle were $145^{\circ}$ and $153^{\circ}$, respectively. To measure residual stress along the depth direction, material removal by electropolishing was used, and the specimen surface was removed by a depth of $0.05 \mathrm{~mm}$ to the top surface every time. After each removal with a depth of $0.2 \mathrm{~mm}$, residual stress values at a regular interval of $0.5 \mathrm{~mm}$ at the center line were recorded. Thus, the surface layer with a thickness of $1 \mathrm{~mm}$ will be removed five times. All measurements were repeated thrice for each point, and the average value was obtained and recorded. According to these experimental data, cross-sectional residual stress distributions at the plane perpendicular to the top surface along the center line can be presented by MATLAB 2010a software developed by The Mathworks in Natick, MA, USA.

After LSP treatment, the as-machined specimen and three types of LSPed specimens used for metallographic investigation were cut as the sections perpendicular to the specimen surface, and then subjected to several successive steps of grinding and polishing. After that, the vertical sections of the samples were etched using a professional reagent that consists of $15 \mathrm{cc}$ of $\mathrm{HCl}, 10 \mathrm{cc}$ of $\mathrm{HNO}_{3}, 10 \mathrm{cc}$ 
of acetic acid, and 2/3 (two thirds) drops glycerine, and then characterized by cross-sectional OM observations. Cross-sectional thin foils for TEM observations were also prepared, and microstructure in the top surface of all specimens was observed by a JEM-2100 transmission electron microscopy (TEM) produced by JEOL, Tonkin, Japan operated at a voltage of $200 \mathrm{kV}$.

\section{Result and Discussions}

\subsection{Surface Residual Stress Distribution at a Single Laser Spot}

Figure 2 shows surface residual stress three-dimensionally displayed distribution at a single laser spot as a function of the number of LSP impacts. For the above three cases, we choose a diameter of every laser spot, as shown in Line 1 in Figure 2a, Line 2 in Figure 2b, and Line 3 in Figure 2c. Figure 3 shows surface residual stress distributions along the diameters of laser spots subjected to single LSP impact, dual LSP impacts, and triple LSP impacts, respectively.

From Figures 2a and 3, it can be seen that there is a maximum of $-239 \mathrm{MPa}$ in residual stress at the center of the laser spot subjected to a single LSP impact. The diameter of the LSP-affected region is about $4 \mathrm{~mm}$. After dual LSP impacts, the maximum of $-239 \mathrm{MPa}$ is increased to $-336 \mathrm{MPa}$, and the center region with a radius of $\sim 0.8 \mathrm{~mm}$ exists in high-level compressive residual stress of more than $-300 \mathrm{MPa}$. The LSP-affected region keeps an increase to a diameter of approximately $4.5 \mathrm{~mm}$, as shown in Figures $2 \mathrm{~b}$ and 3. After a third LSP impact, the maximum of surface residual stress is increased to $-371 \mathrm{MPa}$, and the center region with a radius of $\sim 1.3 \mathrm{~mm}$ exists in high-level compressive residual stress of more than $-300 \mathrm{MPa}$. The LSP-affected region continues to increase to a diameter of approximately $5 \mathrm{~mm}$, as shown in Figures 2c and 3.
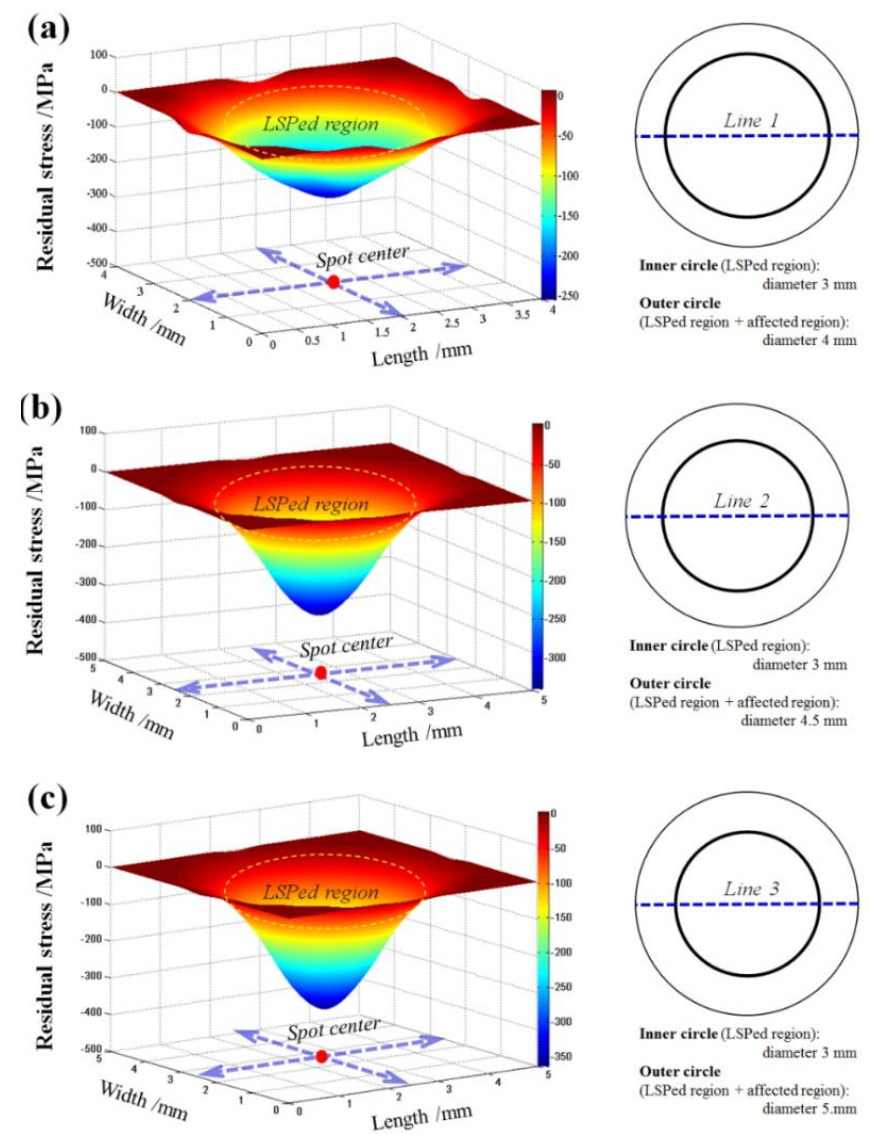

Figure 2. Surface residual stress three-dimensionally displayed distributions at a single laser spot as a function of the number of LSP impacts. (a) Single LSP impact; (b) dual LSP impacts; and (c) triple LSP impacts. 


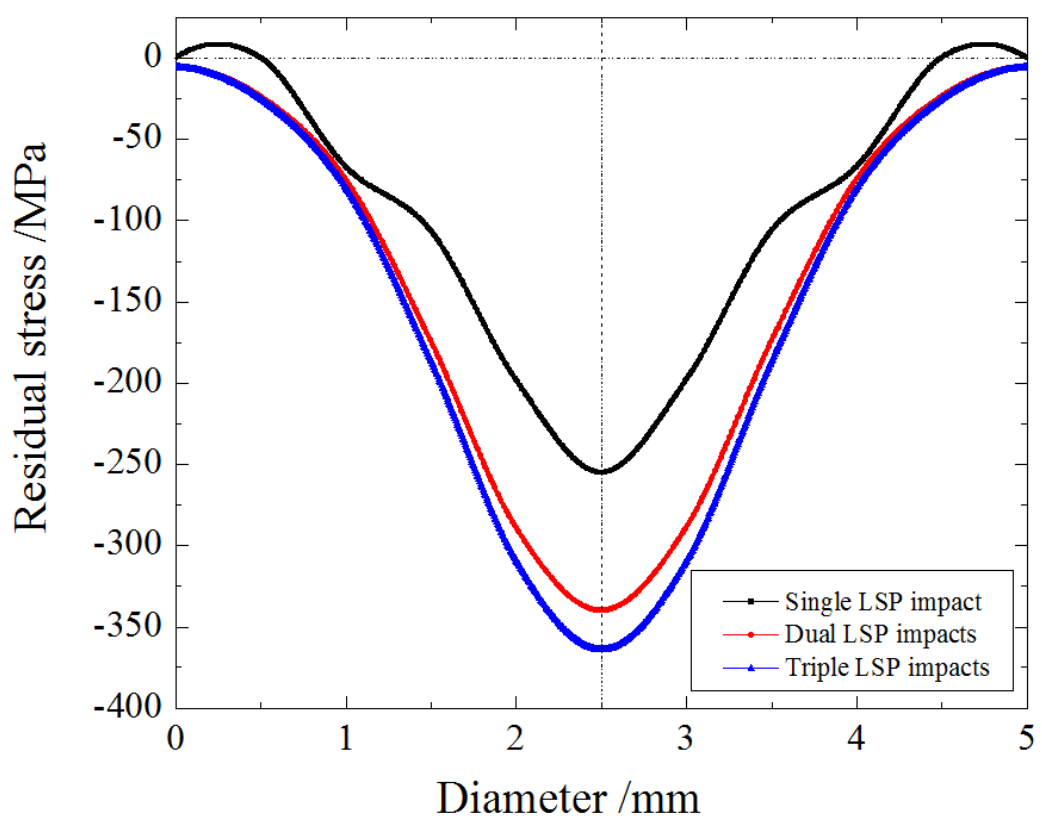

Figure 3. Surface residual stress distributions along the diameters of laser spots subjected to different LSP impacts.

From the above experimental data, the following two facts can be obtained. On the one hand, the maximum of compressive residual stress increases with the increasing number of LSP impacts, but the increasing rate decreases with the increasing number of LSP impacts. On the other hand, while the number of LSP impacts increases, the LSP-affected region increases. In addition, there is only a little change in the LSP-affected region when the number of LSP impacts varies from two to three. We may speculate that the LSP-affected region can reach a peak with the increasing number of LSP impacts. Among the fringe field of the LSP-affected region, the recoiling pressure wave decreases steeply and it cannot reach the yield limit of AISI 304 austenitic stainless steel, which leads to the inadequate plastic deformation of the region far away from the spot center. Hence, surface residual stress along the diameter of the laser spot also decreases gradually with the increasing distance to the spot center. It is worth noting that multiple LSP impacts cause more and more uneven surface residual stress distribution at a laser spot with an increasing number of LSP impacts.

\subsection{Residual Stress Distribution in Depth Direction along the Diameter of Laser Spot}

Figure 4 shows in-depth residual stress three-dimensionally displayed distributions at Line 1 in Figure 2a, Line 2 in Figure 2b, and Line 3 in Figure 2c. Figure 5 shows in-depth residual stress distributions of the spot center subjected to single LSP impact, dual LSP impacts, and triple LSP impacts, respectively. From Figure $4 \mathrm{a}-\mathrm{c}$, in-depth residual stress distributions at the diameter as a function of the number of LSP impacts can be clearly presented. After single LSP impact, the residual stress of the spot center is $-239 \mathrm{MPa}$, and the affected depth of the compressive residual stress is about $0.78 \mathrm{~mm}$, as shown in Figure 5. After dual LSP impacts, the residual stress of the spot center is increased to $-336 \mathrm{MPa}$, and the affected depth of the compressive residual stress reaches $1.05 \mathrm{~mm}$, as shown in Figure 5. After triple LSP impacts, the residual stress value of the spot center and its affected depth are $-371 \mathrm{MPa}$ and $1.18 \mathrm{~mm}$, as shown in Figure 5 . 

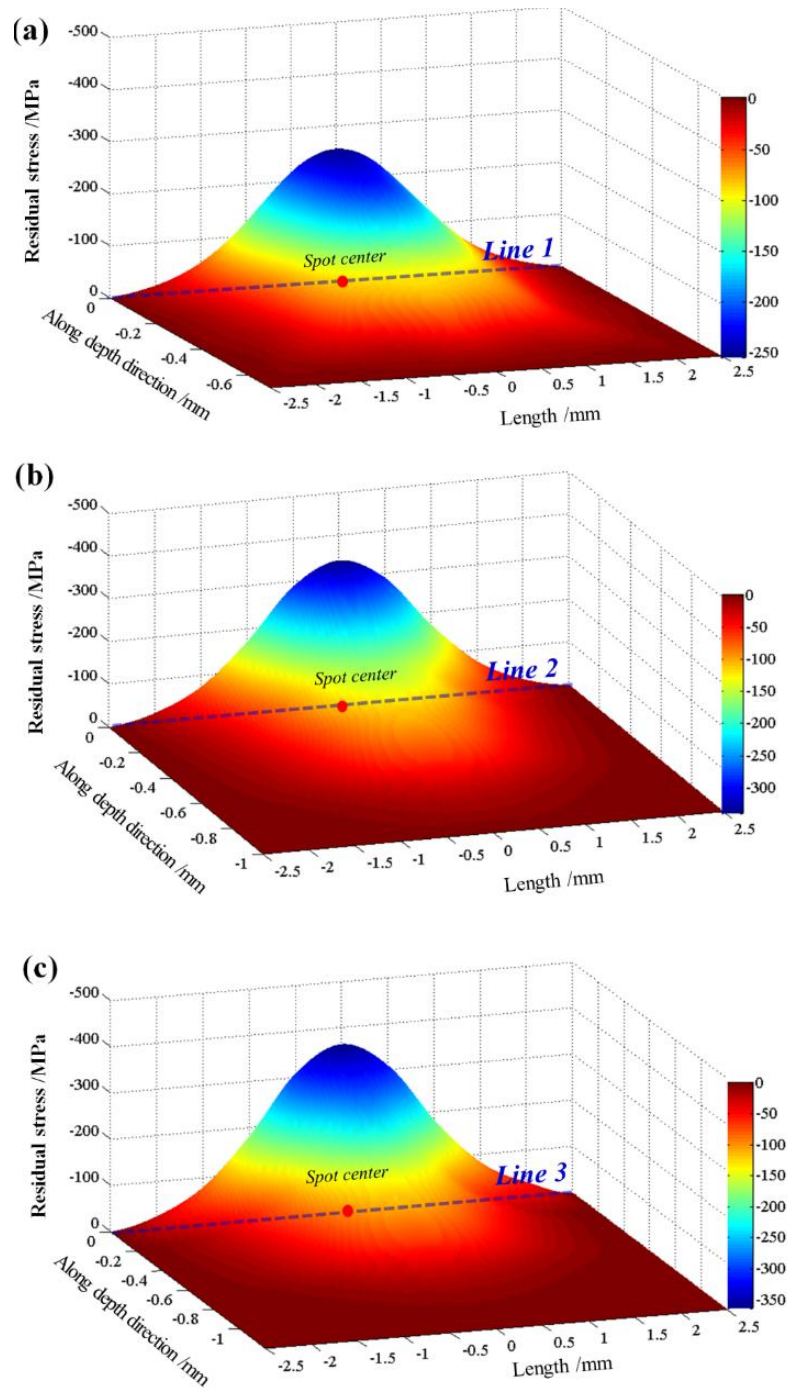

Figure 4. In-depth residual stress three-dimensionally displayed distributions along the diameter of laser spots with different LSP impacts. (a) Single LSP impact; (b) dual LSP impacts; and (c) triple LSP impacts.

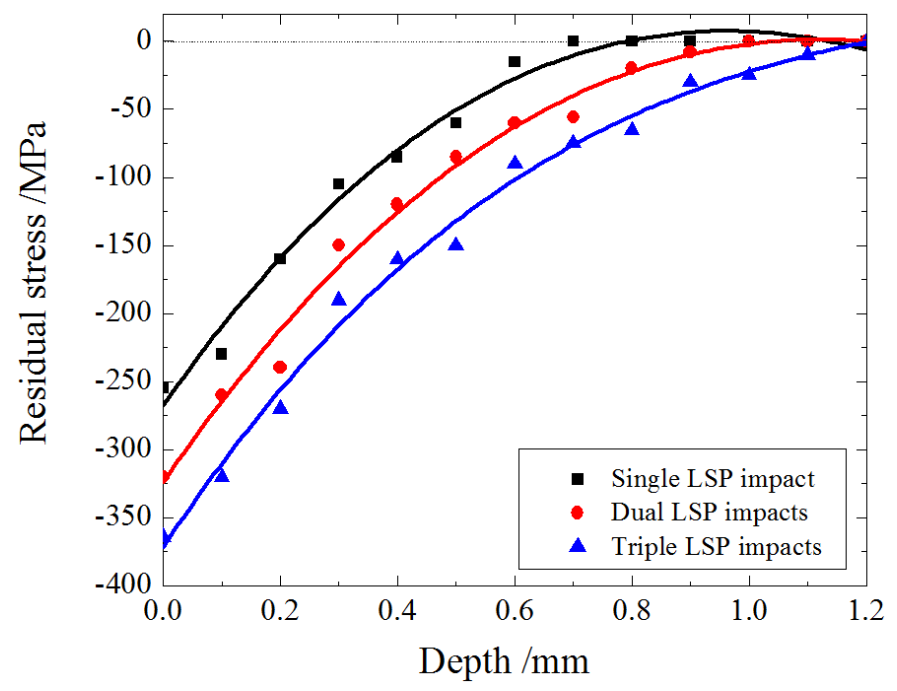

Figure 5. In-depth residual stress distributions of the spot center subjected to different LSP impacts. 
From the above experimental data, it can be concluded that after triple LSP impacts, the residual stress value of the spot center and the affected depth reach a peak, and the compressive residual stress keeps a constant and the affected depth also reaches a saturated value.

\subsection{Metallographic Observation and Microstructural TEM Characterization}

Metallographic observation of the top surface of the as-machined specimen and three types of LSPed specimens can be seen in Figure 6. Figure 7 shows typical TEM images of the top surface of the as-machined specimen and LSPed specimens. The grain size, grain boundary, and grain shape can be recognized after being immersed in the professional etching reagent for $30 \mathrm{~s}$ at room temperature.
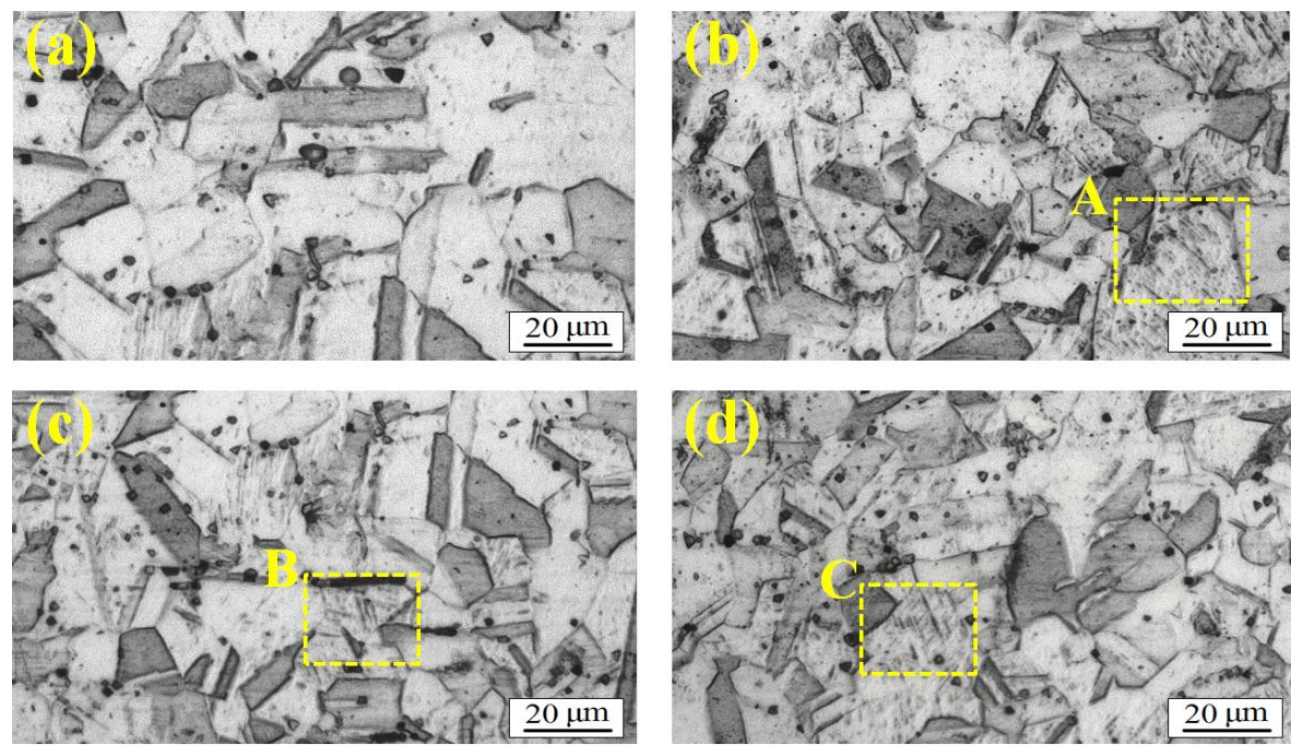

Figure 6. Typical metallographic observations in the top surface of as-machined specimen and three types of LSPed specimens. (a) As-machined specimen; (b) single LSP impact; (c) dual LSP impacts; and (d) triple LSP impacts.
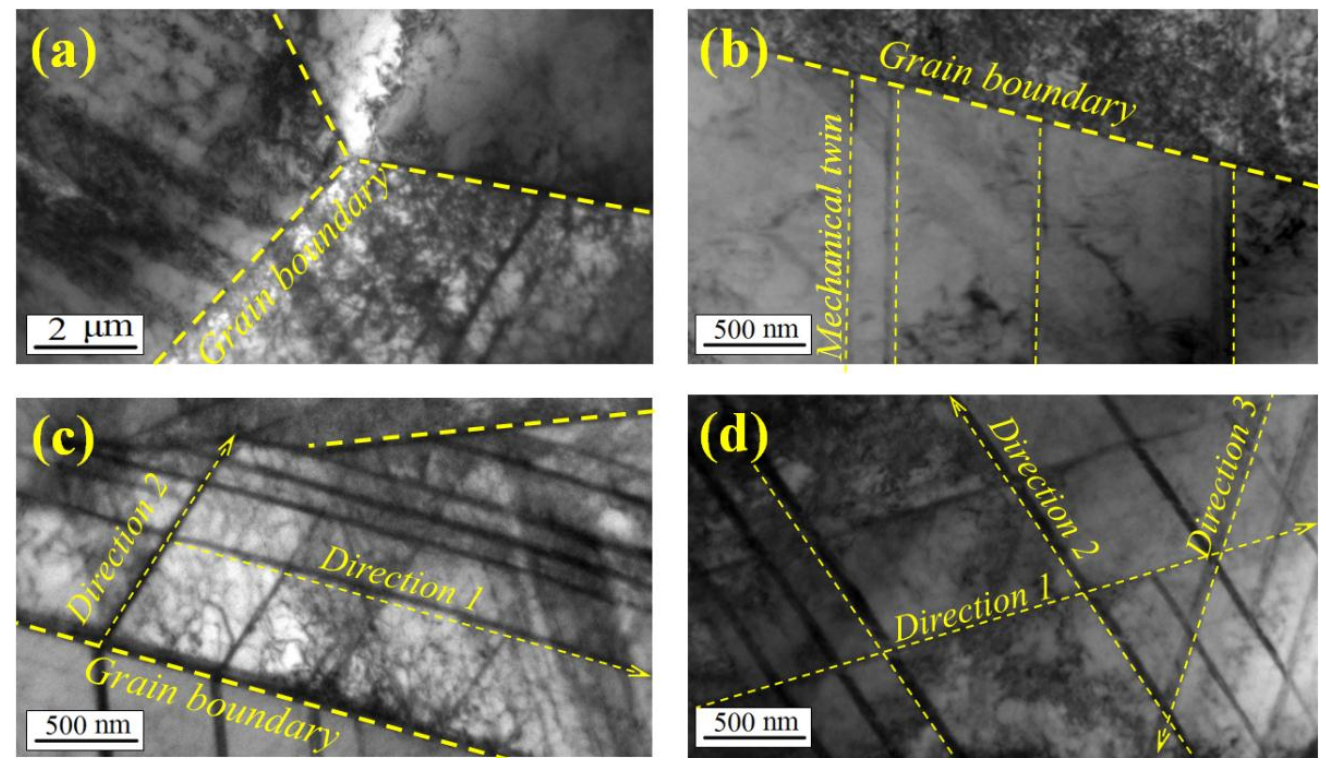

Figure 7. Typical TEM microstructures in the top surface of as-machined specimen and three types of LSPed specimens. (a) As-machined specimen; (b) single LSP impact; (c) dual LSP impacts; and (d) triple LSP impacts. 
From Figure 6a, it can be seen that the original coarse grain in the top surface of the as-machined specimen varies from 20 to $60 \mu \mathrm{m}$. For three types of LSPed specimens, evidence of plastic deformation is obvious in the surface layer, as shown in Figure 6b-d. After a single LSP impact, an overwhelming majority of coarse grains are obviously refined into grains with an average size of 10-20 $\mu \mathrm{m}$, as shown in Figure $6 \mathrm{~b}$. Some coarse grains are not refined, but some mechanical twins (MTs) with one direction can be found within the coarse grain. These MT boundaries are parallel to each other and they subdivide the coarse grain into thin twin-matrix (T-M) lamellae at the top surface, whose width ranges from $40 \mathrm{~nm}$ to $700 \mathrm{~nm}$, as can be clearly seen in Rectangle (A) in Figures $6 \mathrm{~b}$ and $7 \mathrm{~b}$. After a second LSP impact, laser shock wave causes the presence of refined grain with an average size of 5-15 $\mu \mathrm{m}$ (as shown in Figure 6c), and some MTs with two directions can be found within these grains. Moreover, the intersections of MT-MT in two directions' result in submicron rhombic blocks and the average dimension of the submicron rhombic block is about $100 \mathrm{~nm} \times 500 \mathrm{~nm}$, as presented in Rectangle (B) in Figures 6c and 7c. After triple LSP impacts, the refined grain turns more uniform and smaller, and some MTs with three directions can be found within these grains. Under the effect of the third impact, submicron rhombic blocks are transformed into triangular blocks with the dimension of about $130 \mathrm{~nm} \times 130 \mathrm{~nm}$, as shown in Rectangle (C) in Figures $6 \mathrm{~d}$ and $7 \mathrm{~d}$. Hence, it can be concluded that when the number of LSP impacts varies from one to three, the coarse grains are refined by T-M lamellae, submicron rhombic blocks and triangular blocks, respectively.

According to the previous results, there are two reasons that are responsible for the observed MTs of Face-centered cubic (FCC) metals. The first one is small grain size. With the decrease of grain size, the energy needed to form MTs and stacking faults gradually becomes closer to the energy needed to nucleate a perfect dislocation, so the deformation mechanism controlled by normal slip transforms to that controlled by partial dislocation activity [14]. The other important reason is the ultra-high strain rate. With the increase of the strain rate, dislocations align themselves into walls at the twin boundary, which transform submicron subdivided blocks into subgrain boundaries gradually. When the strain rate is further increased, subgrain boundaries are turned by dislocations and transform into equiaxed refined grains $[15,16]$. The dislocation-slip process is suppressed when the strain rate is enough high, assisting the formation of deformation MTs [17,18].

The strain rate of metallic materials and alloys exceeds $10^{6} \mathrm{~s}^{-1}$ during LSP treatment, and refined grains are generated in the top surface. As a result of the grain refinement and ultra-high strain rate, the LSPed region is strengthened according to classical dislocation theory [19]. Moreover, for 304 stainless steel, the grains in the top surface turn smaller and smaller with the increasing number of LSP impacts. The reaction between the laser shock wave and the specimen will be generated in the top surface, leading to the generation of the dislocation and micro-structural deformation (such as MT), which can be explained by the fact that high-level compressive residual stresses are generated in the top surface, and the magnitude of the compressive residual stress in the top surface gradually increases with increasing number of LSP impacts.

The beneficial effect brought by LSP treatment is derived from the generation of a compressive residual stress field and the refined microstructure in the near-surface region at a laser spot. Although the output beam of a single laser pulse exists in flat-top shape (as shown in Figure 1), the uneven distribution of surface residual stress induced by the inhomogeneous deformation at a laser spot is further aggravated when AISI 304 stainless steel is treated by multiple LSP impacts, as discussed in Section 3.1. There is a similar result in in-depth residual stress distribution at a laser spot as a function of the number of LSP impacts, as discussed in Section 3.2. The above fact decreases the effect of multiple LSP impacts. As can be seen in Figure 7d, novel structures with submicron triangular blocks are found at the top surface subjected to three LSP impacts, which is the direct reason of the improvement of the residual stress, nano-hardness, and elastic modulus of AISI 304 stainless steel. Hence, improving the overlapping rate during laser beam sweeping can increase the LSP impact number at a laser spot. When the laser impact overlapping rate is $50 \%$, most of the spots 
of the treated surface are impacted by laser pulse at least three times. Large numbers of submicron triangular blocks are generated on the top surface of AISI 304 stainless steel and the microstructures become uniform. The residual stress, nano-hardness, and elastic modulus on the top surface of AISI 304 stainless steel are greatly improved and distributed uniformly on the extended surfaces. In engineering applications, the surfaces of metallic components are often overlappingly treated by multiple LSP treatments. For future investigation, the uniformity of the residual stress distribution induced by massive LSP treatment will be in a very important position.

\section{Conclusions}

The effects of different LSP impacts on surface and in-depth residual stress distributions and microstructure of AISI 304 stainless steel at a laser spot were investigated, and microstructure in the top surface under different LSP impacts was characterized and analyzed. Some important conclusions can be drawn as follows:

(1) The maximum of the surface compressive residual stress at a laser spot increases with the increasing number of LSP impacts, but the increasing rate decreases with the increasing number of LSP impacts. Meanwhile, the LSP-affected region can reach a peak with the increasing number of LSP impacts. Multiple LSP impacts cause more and more uneven surface residual stress distribution at a laser spot with an increasing number of LSP impacts.

(2) After triple LSP impacts, the residual stress value of the spot center and the affected depth reach a peak, and the compressive residual stress remains constant and the affected depth also reaches a saturated value.

(3) When the number of LSP impacts varies from one to three, the coarse grains in the top surface of AISI 304 stainless steel are refined by three types of substructures: T-M lamellae, submicron rhombic blocks and triangular blocks, respectively.

(4) The underlying influence mechanism of typical substructure induced by laser shock wave on residual stress distribution at a laser spot was revealed. Refined grain and ultra-high strain rate are two important factors to generate high-level compressive residual stresses in the top surface of AISI 304 stainless steel.

(5) Most of the spots of the treated surface are impacted by laser pulse at least three times when the laser impact overlapping rate is $50 \%$. Large numbers of submicron triangular blocks are generated on the top surface of AISI 304 stainless steel and microstructures become uniform. The residual stress, nano-hardness, and elastic modulus are distributed uniformly on the extended surfaces.

Acknowledgments: The authors are grateful for the support provided by National Natural Science Foundation of China (No. 51575242), Natural Science Foundation of Jiangsu Province in China (Nos. BK20140012 and BK20151341), China Postdoctoral Science Special Foundation (No. 2015T80504), and Six Major Talent Peak of Jiangsu Province (2014-ZBZZ-015).

Author Contributions: J.L., W.Z. and K.L. conceived and designed the experiments; W.Z. performed the experiments; J.L. and W.Z. analyzed the data; K.L. contributed reagents/materials/analysis tools; W.Z. and J.L. wrote the paper.

Conflicts of Interest: The authors declare no conflict of interest.

\section{References}

1. Ye, C.; Suslov, S.; Fei, X.L.; Cheng, G.J. Bimodal nanocrystallization of NiTi shape memory alloy by laser shock peening and post-deformation annealing. Acta Mater. 2011, 59, 7219-7227. [CrossRef]

2. Trdan, U.; Grum, J. SEM/EDS characterization of laser shock peening effect on localized corrosion of $\mathrm{Al}$ alloy in a near natural chloride environment. Corros. Sci. 2014, 82, 328-338. [CrossRef]

3. Trdan, U.; Grum, J. Evaluation of corrosion resistance of AA6082-T651 aluminium alloy after laser shock peening by means of cyclic polarisation and ElS methods. Corros. Sci. 2012, 59, 324-333. [CrossRef] 
4. Wang, J.T.; Zhang, Y.K.; Chen, J.F.; Zhou, J.Y.; Ge, M.Z.; Lu, Y.L.; Li, X.L. Effects of laser shock peening on stress corrosion behavior of 7075 aluminum alloy laser welded joints. Mater. Sci. Eng. A 2015, 647, 7-14. [CrossRef]

5. Trdan, U.; Porro, J.A.; José, L.; Ocaña, J.G. Laser shock peening without absorbent coating (LSPwC) effect on 3D surface topography and mechanical properties of 6082-T651 Al alloy. Surf. Coat. Technol. 2012, 208, 109-116. [CrossRef]

6. Jia, W.J.; Hong, Q.; Zhao, H.Z.; Li, L.; Han, D. Effect of laser shock peening on the mechanical properties of a near- $\alpha$ titanium alloy. Mater. Sci. Eng. A 2014, 606, 354-359. [CrossRef]

7. Correa, C.; de Lara, L.R.; Díaz, M.; Porro, J.A.; García-Beltrán, A.; Ocaña, J.L. Influence of pulse sequence and edge material effect on fatigue life of Al2024-T351 specimens treated by laser shock processing. Int. J. Fatigue 2015, 70, 196-204. [CrossRef]

8. Lu, J.Z.; Luo, K.Y.; Yang, D.K.; Cheng, X.N.; Hu, J.L.; Dai, F.Z.; Qi, H.; Zhang, L.; Zhong, J.S.; Wang, Q.W.; Zhang, Y.K. Effects of laser peening on stress corrosion cracking (SCC) of ANSI 304 austenitic stainless steel. Corros. Sci. 2012, 60, 145-152. [CrossRef]

9. Lu, J.Z.; Luo, K.Y.; Zhang, Y.K.; Cui, C.Y.; Sun, G.F.; Zhou, J.Z.; Zhang, L.; You, J.; Chen, K.M.; Zhong, J.W. Grain refinement of LY2 aluminum alloy induced by ultra-high plastic strain during multiple laser shock processing impacts. Acta Mater. 2010, 58, 3984-3994. [CrossRef]

10. Lu, J.Z.; Luo, K.Y.; Zhang, Y.K.; Sun, G.F.; Gu, Y.Y.; Zhou, J.Z.; Ren, X.D.; Zhang, X.C.; Zhang, L.F.; Chen, K.M.; et al. Grain refinement mechanism of multiple laser shock processing impacts on ANSI 304 stainless steel. Acta Mater. 2010, 58, 5354-5362. [CrossRef]

11. Zhan, K.; Jiang, C.H.; Ji, V. Uniformity of residual stress distribution on the surface of S30432 austenitic stainless steel by different shot peening processes. Mater. Lett. 2013, 99, 61-64. [CrossRef]

12. Nikitin, I.; Altenberger, I. Comparison of the fatigue behavior and residual stress stability of laser-shock peened and deep rolled austenitic stainless steel AISI 304 in the temperature range $25-600{ }^{\circ} \mathrm{C}$. Mater. Sci. Eng. A 2007, 465, 176-178. [CrossRef]

13. Withers, P.; Bhadeshia, H. Residual stress Part 2-Nature and origins. Mater. Sci. Technol. 2001, 17, 65-355. [CrossRef]

14. Li, J.; Liao, Y.L.; Suslov, S.; Cheng, G.J. Laser shock-based platform for controllable forming of nanowires. Nano Lett. 2012, 12, 3224-3230. [CrossRef] [PubMed]

15. Chu, J.P.; Rigsbee, J.M.; Banas, G.; Elsayed-Ali, H.E. Laser shock processing effects on surface microstructure and mechanical properties of low carbon steel. Mater. Sci. Eng. A 1999, 260, 260-268. [CrossRef]

16. Tao, N.R.; Wang, Z.B.; Tong, W.P.; Sui, M.L.; Lu, J.; Lu, K. An investigation of surface Nano crystallization mechanism in Fe induced by surface mechanical attrition treatment. Acta Mater. 2002, 50, 4603-4616. [CrossRef]

17. Bohn, R.; Haubopld, T.; Birringer, R.; Gleiter, H. Nanocrystalline intermetallic compounds-An approach to ductility? Scr. Metall. Mater. 1991, 25, 811-816. [CrossRef]

18. Lu, K.; Wang, J.T.; Wei, W.D. A new method for synthesizing nanocrystalline alloys. J. Appl. Phys. 1991, 69, 522-531. [CrossRef]

19. Chen, M.W.; Ma, E.; Hemke, K.J.; Sheng, H.W.; Wang, Y.M.; Cheng, X.M. Deformation twinning in nanocrystalline aluminum. Science 2003, 300, 1275-1277. [CrossRef] [PubMed]

(C) 2015 by the authors; licensee MDPI, Basel, Switzerland. This article is an open access article distributed under the terms and conditions of the Creative Commons by Attribution (CC-BY) license (http://creativecommons.org/licenses/by/4.0/). 